\title{
Multi-criteria decision making approach as a tool for street network re- design in the city of Bitola, North Macedonia
}

\author{
Jasmina Bunevska Talevska ${ }^{a^{*}}$, Marija Malenkovska Todorova ${ }^{a}$ \\ a University St.Kliment Ohridski Bitola, Faculty of Technical Sciences Engineering
}

\begin{tabular}{l} 
A R T I C L E I N F O \\
\hline DOI: 10.31075/PIS.65.02.01 \\
Professional paper \\
Received:15/01/2019 \\
Revised: 22/05/2019 \\
Accepted:02/06/2019 \\
\hline${ }^{*}$ Corresponding author: \\
jasmina.bunevska@tfb.uklo.edu.mk \\
\hline Keywords: \\
Sustainable urban mobility \\
Street re/design \\
MCDM
\end{tabular}

\begin{abstract}
A B S T R A C T
The current urban planning principles are based on the renewal and use of the cities' available potentials, with the aim of their sustainable urban mobility development. Today, according to the literature, there is no doubt that urban reconstruction is a much better direction for the development of smart cities since numerous challenges threaten the ability of cities to become viable pillars of sustainable development. The main objective of this paper is to adopt recently developed methodology for the analysis and selection of pedestrian crossing types on the case study location example regarding first phase on sustainable urban mobility plan development for the Municipality of Bitola, North Macedonia, as well as to underline the principles and assessment procedure for the re-design of urban network in Bitola as a sustainable urban form.
\end{abstract}

\section{Introduction}

Pedestrians and bicyclists are among the most vulnerable road users. In many countries, collisions with pedestrians are a leading cause of death and injury, and over half of all road deaths are caused by collisions between vehicles and pedestrians, that occur in a number of situations, especially including walking while trying to cross the road. Road traffic crashes kill 1.25 million people every year, almost $90 \%$ of road traffic deaths occur in low- and middle-income countries, road traffic injuries are the \#1 cause of death for people aged 15-29 years, road traffic crashes cost countries around $3 \%$ of their GDP. Moreover, according to Principle 2 of the Declaration of the Rights of the Child, "The child shall enjoy special protection, and shall be given opportunities and facilities, by law and by other means, to enable him/her to develop physically, mentally, morally, spiritually and socially in a healthy and normal manner and in conditions of freedom and dignity"[1].

Since the current urban planning principles are based on the renewal and use of the cities' available potentials, with the aim of their sustainable development, the general aim of our work is to emphasized that SUMP (Sustainable Urban Mobility Plan) components: safety and security are a core components in creating sustainable urban mobility, and are primary areas of action, particularly in making roads safer and more secure for 'vulnerable' road users. Improving the safety and security of modes can be an extremely important step in encouraging users to change (or even try) alternative modes, especially when they are perceived as 'unsafe'. One of the urban mobility measures and management is traffic calming. It is a combination of a plurality of physical measures, which aim to reduce the negative effects of the use of motor vehicles, bringing to change driver behavior and reduce the maximum speed of which would achieve greater traffic safety. Traffic calming is defined as a concept of transport policy that includes promotion of traffic to pedestrians, cyclists and public transport, and a reduction in average speeds for vehicles in residential areas.

\section{City of Bitola toward sustainable urban mobility}

"Sustainable urban mobility" also known as shortdistance mobility of individuals and non-motorized transport as walking and cycling within the Bitola is impaired. City has become more focused on the transportation of vehicles, as use of cars has increased, than on the ability of people to enjoy the space [2]. This has led to city streets that function poorly in their ability to move traffic. Drivers are focused on traffic signals and signage and rarely communicate either visually or verbally with pedestrians along the road. When drivers are not at stoplights, they are often going 
as fast as the posted limit will allow, which further distances the drivers from pedestrians who are looking to cross the road safely. For the support of "Local mobility", key elements are the pedestrian and bicycle friendly site and street design and a mixture of land uses combined with parks and green spaces. Having in mind that nearly every trip for example by public transport or by car, starts and ends with a walking distance, the significance of "Local mobility" is even higher. And at last but not least, we need to keep in mind that the dependence of children or elderly people on nonmotorized traffic increases the significance of "Sustainable urban mobility".

\subsection{Traffic calming}

Traffic engineers, public works officials, and urban planners have many strategies for traffic calming including speed humps or tables, emergency-friendly speed cushions, lane narrowing, traffic circles, and improved bicycle access with designated bike lanes. These solutions are used to create more complete streets that offer safe access to all users.

Traffic calming has been shown to:

- Reduce speeding;

- Improve speed limit compliance;

- Decrease injury severity when crashes occur;

- Lower fatality rates;

- Encourage varied transportation modes;

- Improve street aesthetics;

- Increase property value;

- Reduce crime.

While solutions can be instituted at the street, neighborhood, or city-wide level, traffic calming is most effective when it is part of a proactive program to improve street safety based on highest need. This is often ascertained with a points program to determine where risk is highest.

\section{Multi-criteria aproach}

Since 1957 numerous methods for conducting multicriteria ranking have been developed. Some of them are: SAW (Simple Additive Weighting Method), TOPSIS (The Technique for Order of Preference by Similarity to Ideal Solution), ELECTRA (Elimination Et Choix Traduinsant Realite), PROMETHEE (A Preference Ranking Organization Method for Enrichment Evaluation), VIKOR, AHP (Analytic Hierarchy Process), etc [3-5].

Recently developed multi-criteria decision-making analysis based on the AHP - Analytic Hierarchy Process approach synthesizes the aspects of different opinions by weighing up many subjective factors and which studies the unique common result [6]. The level of consistency allows us to form an adjustment of judgments. At the end of the process we answered how to best make a decision in a complex, subjective situation with more than a few realistic options. Namely, for the application of multicriteria decision method we set the goal, i.e. alternatives, adequate number of criteria and sub criteria for precise ranking of the alternative, as in (Table 1).

Table 1. Decision making alternatives and criterions

$$
\text { C1 C2 ......... Cn }
$$

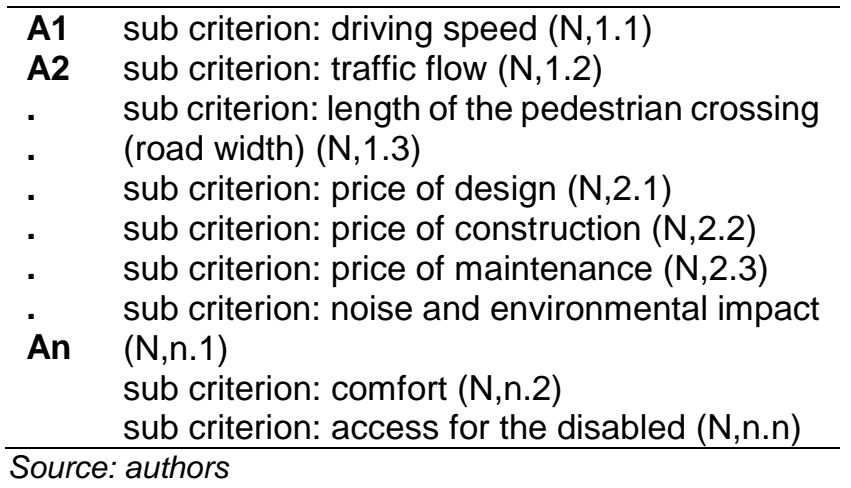

The main objective of this analysis is to use recently developed methodology regarding the selection of the most suitable pedestrian crossing type for the city of Bitola, North Macedonia, and the most important aim is directed towards obtaining pedestrian and bicyclist safety, bearing in mind the role of pedestrian safety within the current safety goals [3].

\subsection{Decision hierarchy criterions and sub- criterions}

Safety is a condition in which a pedestrian can normally cross a pedestrian crossing, with the process not being disturbed nor degraded due to various threats and dangers.

Driving speed has been identified as a key risk factor in road traffic injuries, influencing both the risk of a road crash as well as the severity of the injuries that result from crashes. Excess speed is defined as exceeding the speed limit. In inappropriate speed, the pedestrian cannot properly estimate the moment at which the vehicle will reach the pedestrian crossing i.e. the point of intersection between the paths of the vehicle and the pedestrian, and the motorist is not able to stop the vehicle on time. The greater the difference in the speed between the pedestrian and the vehicle, the greater the danger to the pedestrian.

If traffic flow saturation results in situations in which the time gap between the approaches of two succeeding vehicles is shorter than the time required crossing the road, the method of stopping the vehicle has to be applied in order to perform the crossing. If traffic is of a higher intensity resulting in even scarcer occurrences of suitable intervals to cross the road, the pedestrians lose 
patience and recklessly step onto the roadway. The consequences of such actions may be catastrophic and in such situations zebra crossings do not usually match the needs and a signalized crossing needs to be constructed. Should traffic lights cause very long queues of vehicles, and pedestrian waiting time exceed the limit of patient waiting, then the pedestrian crossings are grade-separated, by constructing overpasses.

The length of the pedestrian crossing is in correlation with traffic safety. The crossing time using a longer pedestrian crossing means longer stay of the pedestrian on the roadway and higher risk of getting injured. On a multi-lane road, the vehicles moving along the right curb often obscure the view of vehicles that move along the farther lane. This phenomenon is especially noted in cases when small children want to cross the street and the motorists fail to notice them on time. This problem is especially emphasized in the vicinity of schools.

Price is the value that is put to a product or service and is the result of a complex set of calculations, research and understanding and risk taking ability. A pricing strategy takes into account segments, ability to pay, market conditions, competitor actions, trade margins and input costs, amongst others. It is targeted at the defined customers and against competitors. In forming the criteria of prices for pedestrian facilities, costs of design, construction, and maintenance, have been taken into consideration and studied as separate sub criteria.Bearing in mind that there are no exact numerical indicators the criterion environment \& comfort serves as an additional assistance to the decisionmakers.

Exposure to noise, in everyday urban life is considered to be an environmental stressor. A specific outcome of reactions to environmental stress is a fast pace of life that also includes a faster pedestrian walking speed. On zebra crossings and signalized crossings pedestrians are exposed at great noise level, whereas in underpasses and overpasses they are much better protected. The influence of noise is greater the closer the vehicles are to the pedestrian crossing and the larger their number. This influence is most expressed in peak hours when the highest number of vehicles is on the road, as well as pedestrians.

The sub criterion aesthetic and environment considers the negative impacts of pedestrian crossing construction on the environment, changes of the streetscape, unpleasant experiences as well as a feeling of personal protection.

The concept of accessible design for disabled persons ensures both direct access, i.e. unassisted, and indirect access, that is compatibility with a person's assistive technology (for example, computer screen readers). This is about making everything accessible to all people, whether they have a disability or not.

\section{Environmental impact of street re/design}

Traffic have negative impacts on the environment. As it is pointed in the literature urban transport is responsible for almost $40 \%$ of $\mathrm{CO}_{2}$ emissions and nearly $70 \%$ of other produced pollutants. The high concentration of cars and the resulting congestion contribute to this global trend. Urban traffic is responsible for more than $10 \%$ of total EU emissions.

The main objectives of environmental protection are:

- Reduce regional pollution responsible for today's poor air quality: Particulate Matter (PM10), NOx and $\mathrm{SO}_{2}$ emissions are addressed here;

- Reduce the global climate change and the greenhouse effect: $\mathrm{CO}_{2}$ emissions;

- Reduce $\mathrm{SO}_{2}, \mathrm{NOx}$ and $\mathrm{NH}_{3}$ transport's emissions, which cause the depletion of the ozone layer;

- Reduce bio-diversity severance and changes on local environment stability.

Even if few street re/design systems have been implemented as environmental measures, many are designed with this objective in mind. In some cases the objective is to reduce the impacts of traffic, but in others the intention is to protect certain areas such as city centers or residential zones. Poor integration of the transport system with the rest of the city has negative impacts on street and outdoor livability.

The objective of livability entails a variety of transport impacts that have already been mentioned as responsible for the poor quality of our cities:

- Reduce local emissions responsible for poor air quality;

- Increase safety, because insecurity create stress while walking, cycling and driving;

- Reduce noise emissions from traffic;

- Improve cleanliness of cities suffering from transport vandalism and damages.

Finally, increasing livability translates into increasing comfort while enjoying a city. This is important for citizens, tourists and visitors. Higher livability levels improve the image of a city. 


\section{Safety impact of street re/design}

Unsafe transport systems generate accidents. The objective of safety is to reduce the number of accidents for all modes, and initiate a decline in the severity level of traffic accidents. Urban transport is responsible for almost $33 \%$ of all fatal accidents involving transport. Even if the number of fatal accidents is on the decline in the EU, the European Commission highlights the need for an even greater decrease. The importance given to safety issues comes from safety's external costs, which confirm the high willingness to pay of citizens to reduce road human damages. Many traffic injuries are directly related to design. Conditions become more dangerous with the addition of speed. Common causes for traffic fatalities include the following:

Lack of Sidewalks: When the sidewalk is blocked, narrow, or nonexistent, pedestrians are forced into the roadbed. This presents a particular threat when the street is designed for fast-moving vehicles, and not designed to accommodate all users safely.

Lack of Accessible Crossings: Pedestrians are at risk of being struck when accessible crossings are not provided or are inaccessible. Mid-block pedestrian crashes are very common on large streets, where vehicle volumes and speeds are prioritized over sufficient opportunities for safe crossing.

Lack of Protection: Wide, multi-lane streets without refuge spaces expose pedestrians to moving vehicles for longer distances as they cross the street. This is particularly unsafe for the elderly or those who move at a slower pace.

Lack of Predictability: When signals and countdown clocks are not provided, or when signal cycle lengths result in a long wait time, pedestrians are unable to safely judge the time they have and are more likely to cross unsafely.

Lack of Cycle Facilities: Cyclists are at risk of rear-end and overtaking crashes when mixing with motor vehicles at moderately high speeds, especially on multi-lane streets.

Poor Intersection Design: Large intersections are often designed for dangerous, high-speed turning. Lack of visibility results in poor navigation and assessment of different users' movements.

Unsafe Boarding Areas: Transit riders are at risk when boarding and alighting vehicles in traffic, especially if no safe facilities are provided. Higher-speed streets and poor intersection design near boarding areas increase chances for severe crashes and put vulnerable users at risk.

Surface Hazards: Obstacles and surface degradation, including potholes, can present hazards to pedestrians and cyclists.

\section{Conclusion}

Crossing a busy street with fast flowing traffic can be very difficult. Pedestrian median islands for example can help pedestrians to cross such roads safely by allowing them to cross in two stages and deal with one direction of traffic flow at a time. They can be used where there is a demand for pedestrians to cross the road, but where the number of pedestrians is not high enough to warrant a signalized pedestrian crossing. Median islands can be part of no-signalized pedestrian crossing, and are usually used on wide, multi-lane roads, in function of narrowing the lanes for vehicular traffic. They must be clearly visible to traffic both day and night.

One effective way of preventing crashes between vehicles and pedestrians is placing them at different levels, or 'grade separating' them. In urban situations where pedestrian crossing signals would cause congestion or crashes (due to high traffic speeds), a grade separated pedestrian crossing, such as an overpass or an underpass, may be used. Outside of urban areas, in situations where there is pedestrian demand in high speed environments, this treatment may also be applied. Grade separated pedestrian crossings reduce pedestrian crashes but they also have some disadvantages: they are costly; pedestrians may avoid them if there are a lot of steps to climb up or down. What is more, if they are not well-lit and patrolled, they may pose a personal security risk. Pedestrians tend only to use crossing facilities located at, or very near, to where they want to cross the road. Where a lot of cycling traffic is present, a pedestrian underpass or overpass can be used by cyclists as well as pedestrians, but this will require shallow approach ramps and therefore additional land.

Street tightening as specific approach, are extensions of the sidewalk or islands on one or both sides of the street, which on this spot narrow street. The expansion may be a footpath or green space with vegetation. An effective result is achieved with a combination of extended walkway and flat hump. The road where the traffic is in two directions, the tightening is performed at 20 feet (6 meters), typically dodges width of 13 to 17 feet (4 to 5 meters).

The effects of street tightening are:

- The speeds will be reduced to $4 \%$ if the tightening is on one side, $14 \%$ if there is a tightening on both sides of the street;

- Reduce the passage of travelers through the wide streets and places to secure better visibility for them;

- Provide better view of the street with greenery on the islands at the ends of the streets. 
Chicanes represent a series of alternating tapers or deformation of the edge that extends from one side to the other side of the street forming an "S" turns. If the narrowing is performed at 10 to $15 \mathrm{~m}$, they can be effective in reducing speed. Apply in places where there is an equivalent flow of vehicles in both directions. Not admitted to the difficulty of maneuvering the buses.

\section{References}

[1] UN General Assembly, (1959).Geneva Declaration, proclaimed by General Assembly Resolution 1386(XIV) of 20 November 1959

[2] Bunevska, T.J., Biljanovski, M.: (2018). City of Bitola toward urban sustainability, Proceedings of the $2^{\text {nd }}$ International Scientific Conference Transport For Today's Society, University of Bitola, Faculty of Technical Sciences, Macedonia

[3] Kuzović, L. T., Aleksić, B. L., \& Glavić, D. N. (2015). The application of the multi-criteria analysis in evaluating of the road designs. Tehnika, 70(1), 143-150.

[4] Glavić, D., Milenković, M., Trpković, A., Vidas, M., \& Mladenović, M. N. (2017). Assessing sustainability of road tolling technologies. International congress on transport infrastructure and systems, Rome.

[5] Milenković, M., Glavić, D., \& Mladenović, M. N. (2018). Decision-support framework for selecting the optimal road toll collection system. Journal of Advanced transportation, 2018.

[6] Bunevska, T.J., Mitev,R., Malenkovska, T.,M., (2018). Development of methodology for the selection of the optimal type of pedestrian crossing, a case study, $2^{\text {nd }}$ International Conference on Management, Engineering and EnvironmentICMNT2018, Serbia. 